\title{
Design of In-phase and Quadrature Two Paths Space-Time-Modulated Metasurfaces
}

\author{
Xinyu Fang, Mengmeng Li, Senior Member, IEEE, Dazhi Ding, Filiberto Bilotti, Fellow, IEEE, Rushan \\ Chen, Senior Member, IEEE
}

\begin{abstract}
Space-time-modulated metasurfaces can manipulate electromagnetic waves in space and frequency domain simultaneously. In this paper, an analytical design of space-timemodulated metasurfaces with modulation elements composed of two paths, In-phase (I) and Quadrature (Q), is proposed. The model is derived analytically, the space/frequency domain manipulations are achieved by designing the dimension and time sequence of $I$ and $Q$ paths. In the specular reflection direction, an objective frequency shift of the reflected first order harmonic can be obtained. While, in other directions, the opposite first order harmonic can be easily controlled by changing the dimension of I/Q paths and the objective first order harmonic remains unchanged. Furthermore, with a small dimension of I/Q paths, the first order harmonic can be used for beam scanning by pre-designing the start time of the modulation element. To realize the space-time-modulated metasurface with the required periodically time-varying responses, 2-bit unit-cells loaded with dynamically switchable pin diodes are employed as I/Q modulation. Both analytical and numerical results demonstrate that space and frequency domain manipulations of the reflected fields by the first order harmonics can be simultaneously obtained. The proposed designs have potential applications in wireless communications, radar camouflaging, and cloaking.
\end{abstract}

Index Terms-Metasurfaces, space-time modulation, analytical design.

\section{INTRODUCTION}

M ETASURFACES have obtained a great attraction for the manipulation of electromagnetic waves, due to their low cost fabrication and thin profile [1]-[3]. They have been widely used for numerous applications, such as beam steering

Manuscript received xxxx. This work was supported in part by Natural Science Foundation of China of 61871222, 62025109, and 61890541, the Fundamental Research Funds for the Central Universities of 30921011101. FB acknowledges the support of the research contract MANTLES funded by the Italian Ministry of University and Research, PRIN 2017 n. 2017BHFZKH.

X. Y. Fang, M. Li, D. Z. Ding, and R. S. Chen are with the Department of Communication Engineering, Nanjing University of Science and Technology, Nanjing, China (e-mail: limengmeng@njust.edu.cn; dzding@njust.edu.cn; eerschen@njust.edu.cn).

Filiberto Bilotti is with the Department of Engineering at ROMA TRE University, Via Vito Volterra 62, 00146 Rome, Italy (filiberto.bilotti@uniroma3.it).
[4]-[7], absorption [8]-[10], focusing [11][13], imaging [14]-[17], etc. To achieve manipulation of the incident electromagnetic fields in the space domain [18]-[20], the design of the reflection/transmission responses of the metasurface at the frequencies of interest are required, and the responses do not change in time. Recently, dynamically reconfigurable metasurfaces can be achieved by using pin diodes [21], [22], varactors [23]-[25], MEMS [26], graphene [27], liquid crystal [28], etc.

To achieve the frequency modulation of the electromagnetic field interacting with a metasurface, the response of the metasurface itself must change in time. A time-modulated reflective metasurface producing a frequency shift with respect to the impinging radiation is proposed in [29]: the reflection phase of the whole metasurface changes linearly with time and an artificial Doppler effect for a non-moving electrically thin structure is realized. In [30], [31] and [32], 2-bit and 3-bit time varying phases are employed to match the linear phase change, respectively, and a frequency shift can be induced for different higher order harmonics. In [33], a transparent, time-modulated metasurface, which functions as a serrodyne frequency translator, is reported at X-band frequencies. With a simple biasing architecture, the metasurface provides electrically tunable transmission phase that covers $360^{\circ}$. A sawtooth waveform is used to modulate the metasurface, allowing Doppler-like frequency translation.. In [34], time modulation engineered asymmetric photonic band gaps are proposed to generate optical isolation.

To manipulate the electromagnetic field in both space and frequency domains, digital space-time-coding metasurfaces have been proposed in [35]. In this case, the equivalent reflection amplitude $A^{m}$ and phase $\psi^{m}$ of the metasurface in frequency domain for the $m t h$ order harmonic frequency $\omega_{c}+$ $m \omega_{0}$ is derived, and a binary particle swarm optimization (BPSO) algorithm is applied to design the space-time-coding sequences. In [36], an $N$-path space-time-modulated metasurface is proposed to manipulate the first order harmonics in space domain using the time delay of each modulation path. The resulting space-time modulated metasurfaces exhibit unprecedented capabilities and may find applications in different operative scenarios, such as non-reciprocal structures [37]-[41], arbitrary multi-bit programmable metasurfaces [42], wireless communications [43]-[48], spread-spectrum radar camouflaging [49], etc.

In this paper, inspired by the concept of time-modulated phased arrays [50], an analytical design model for space-time-modulated metasurfaces with modulation elements composed of two paths, In-phase (I) and Quadrature-phase (Q), is proposed. Using this analytical model, the first order 
harmonics can be manipulated by designing the dimension and the time sequence of the I/Q paths. In the specular reflection direction, an objective frequency shift of the reflected first order harmonic can be obtained. Furthermore, with a small dimension of the I/Q paths, the first order harmonic can be used for beam scanning by pre-designing the start time of the modulation element. In non-specular directions, the opposite first order harmonic is manipulated by changing the dimension of the I/Q paths, keeping the objective first order harmonic unchanged.

An actual implementation of the $\mathrm{I}$ and $\mathrm{Q}$ two paths space-time-modulated metasurface is also proposed, making use of a 2-bit unit-cell exhibiting properly designed time-varying phases as I/Q modulation that can be dynamically switched by using pin diodes. Both analytical and numerical results demonstrate that the space and time modulation of the incident electromagnetic field can be accurately and effectively achieved with the proposed I/Q two paths space-time modulated metasurface.

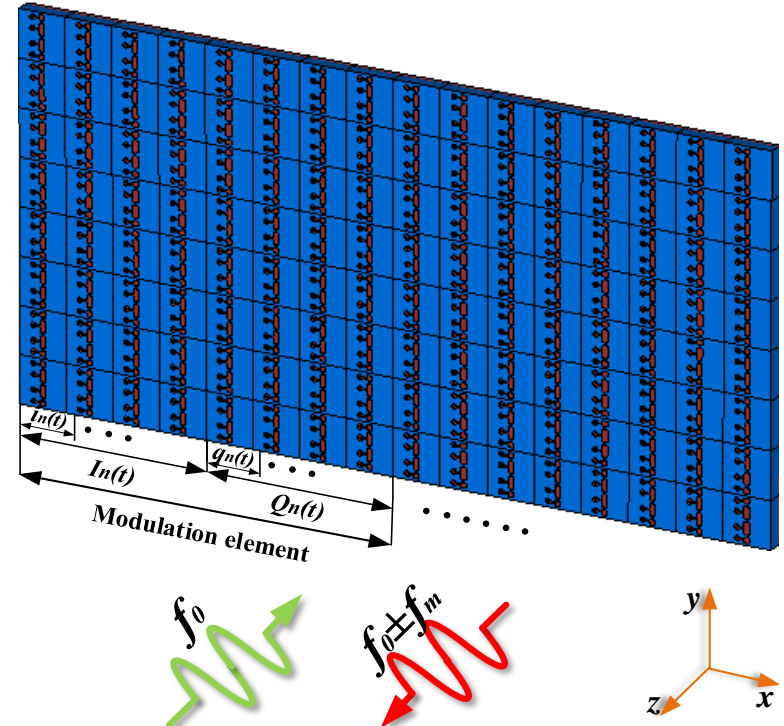

Fig. 1. Geometrical sketh of a metasurface with modulation elements composed of I/Q two paths. The structure is illuminated by a normally impinging electromagnetic wave at frequency $f_{0}$ and propagating in the negative $z$-direction, while the reflected wave is at frequency $f_{0} \pm f_{m}$. The metasurface consists of $N$ modulation elements, each of which is composed of a pair of I/Q paths, made by $N_{s u b}$ unit-cells each. The time sequences of the I/Q of the $n t h$ modulation element are indicated as $I_{n}(t)$ and $Q_{n}(t)$, respectively, while of the time sequences of the unit-cells for each of the two paths are $i_{n}(t)$ and $q_{n}(t)$, respectively.

The paper is organized as follows. In Sec. II, we derive the analytical model to design the I/Q space-time-modulated metasurface. In Sec. III, we present the implementation of the I/Q space-time-modulated metasurface through 2-bit unit-cells exhibiting the required periodically time-varying phases. In Sec. $\mathrm{IV}$, we present the simulation results to verify the effectiveness of the proposed design of the I/Q space-time-modulated metasurface. Finally, in Sec. V, we briefly draw the conclusions of our work.

\section{ANALYTICAL I/Q TWO PATH SPACE-TIME-MODULATED METASURFACE DESIGN}

\section{A. Background of $I / Q$ space-time-modulation}

The main purpose of this paper, is to propose a design method for the I/Q space-time modulated metasurface, as shown in Fig. 1. To this end, we follow the single side band (SSB) modulation method for time-modulated phased antenna arrays proposed in [50]. Without loss of generality, the metasurface is normally illuminated by a plane wave $E_{i}(t)=E_{1} e^{j 2 \pi f_{0} t} ;$ the reflected wave is given by:

$$
E_{r}(t)=E_{i}(t) \cdot \Gamma(t)
$$

where $\Gamma(t)=a(t) e^{j \varphi(t)}$ is the time varying reflection coefficient with amplitude $a(t)$ and phase $\phi(t)$. If we desire a modulated reflected wave as $E_{r}(t)=E_{2} e^{j 2 \pi\left(f_{0} \pm f_{p}\right) t}=E_{2} e^{j 2 \pi f_{0} t} \cdot e^{ \pm j 2 \pi f_{p} t}$ in the specular reflection direction, we have to design the metasurface in such a way that the reflection coefficient $\Gamma(t)$ introduces the frequency shift $e^{ \pm j 2 \pi f_{p} t}$. Considering the positive sign case, we can rewrite the frequency shift in the form of I and Q terms:

$$
\begin{aligned}
e^{j 2 \pi f_{p} t} & =\cos \left(2 \pi f_{p} t\right)+j \sin \left(2 \pi f_{p} t\right) \\
& =\cos \left(2 \pi \frac{t}{T_{p}}\right)+j \sin \left(2 \pi \frac{t}{T_{p}}\right),
\end{aligned}
$$

where $\cos \left(2 \pi \frac{t}{T_{p}}\right)$ denotes the I term, $\sin \left(2 \pi \frac{t}{T_{p}}\right)$ denotes the $\mathrm{Q}$ term, $T_{p}$ is the time period related to $\omega_{p}$. Since $\cos \left(2 \pi \frac{t}{t_{p}}\right)=-\sin \left[2 \pi\left(\frac{t}{t_{p}}-\frac{1}{4}\right)\right]$, eq. (2) can be rewritten as:

$$
e^{j \omega_{p} t}=-\sin \left(2 \pi \frac{t^{\prime}}{T_{p}}\right)+j \sin \left(2 \pi \frac{t}{T_{p}}\right)
$$

where $t^{\prime} / T_{p}=t / T_{p}-1 / 4$. From the right hand part of (3), it is clear that the first term has a quarter-period delay with respect to the second term.

In order to introduce such a frequency shift, we consider the metasurface depicted in Fig.1, where the response of each column (in the $y$-direction) is the same. Then, we group the columns to define a "modulation element" consisting of a pair of I/Q paths, whose time variations of the reflection coefficient follow the two sinusoidal variations in (3) to introduce a frequency shift $f_{p}$ to the reflected field..

The simplest implementation of this modulation scheme consists in adjusting the phase of the two paths according to the rectangular pulse signal whose amplitudes " +1 " and " -1 ", correspond to the phase " 0 " or " $\pi$ ", respectively. In order to maximize the amplitude of the +1 st or -1 st order harmonics and suppress the unwanted higher order harmonics, proper time sequences of the rectangular pulse are needed, as shown in Fig. 2. 


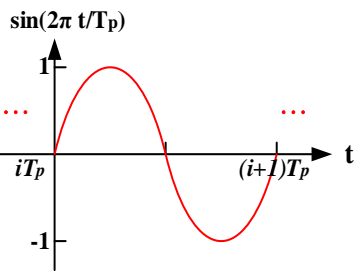

(a)

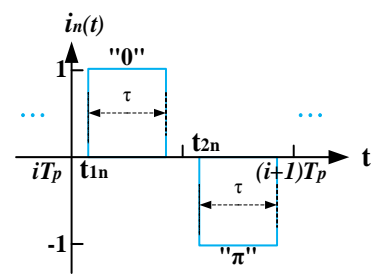

(c)

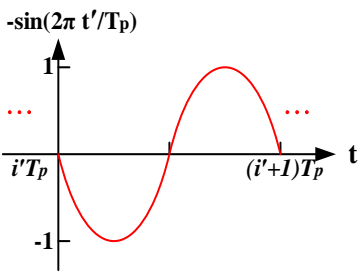

(b)

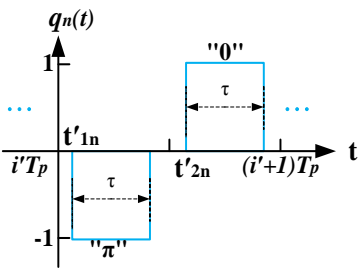

(d)
Fig. 2. Time sequences used to modulate the $n t h$ modulation element. (a) Real part of $e^{j 2 \pi f_{p} t}$, namely I path, (b) imaginary part of $e^{j 2 \pi f_{p} t}$, namely Q path, (c) and (d) rectangular pulses used to match the functions in (a) and (b).

$i_{n}(t)$ and $q_{n}(t)$ are the time sequences of the unit-cells for each of the two paths of the $n t h$ modulation element with period $T_{p}$ matching the two time terms in (3), respectively. $t_{1 n}, t_{2 n}, t_{1 n}^{\prime}$, and $t_{2 n}^{\prime}$ denote the start time of the " 0 " and " $\pi$ " phase states, respectively, being $\tau$ the duration of both phase states. There is no scattering field during the other intervals of the modulation period. Since a " $\pi$ " phase difference between the two paths of a modulation element is needed (see (3) and Fig. 2), and each path has two phase states with a " $\pi$ " phase difference, a 2-bit unit cell is required for the I/Q modulation of the metasurface.

In conclusion, the proposed approach is different from the well-established one based on the linear modulation of the reflection coefficient phase by modulating the single unit-cell element [29]. The latter method, in fact, which requires multi-bit phases to achieve a frequency conversion and is characterized by the same frequency spectra in every observation direction, results in designs based on the time dimension only. On the contrary, our proposed method exploits two paths to realize the I/Q distributions, and each path just needs 1-bit phases. Furthermore, the first order harmonics can be controlled by designing the dimension and the time sequence of the I/Q paths, resulting in designs based on both time and space dimensions.

\section{B. Analytical model of the $I / Q$ space-time modulation element}

By assuming the time-modulation speed much smaller than the plane wave frequency. The field scattered by the I/Q modulated metasurface depicted in Fig 1, can be written using the array factor as:

$$
A F(\theta, t)=e^{j 2 \pi f_{0} t} \sum_{n=1}^{N}\left[I_{n}(t)+j Q_{n}(t) e^{j \beta N_{s b b} p \sin \theta}\right] e^{j \beta(n-1) d \sin \theta}
$$

where $\beta$ is the free space propagation constant at $f_{0}, \theta$ the scattering direction from the metasurafce, $N$ the number of modulation elements, $N_{\text {sub }}$ the number of unit-cells in the path, $p$ the period of the unit-cell, and $d=2 \times N_{\text {sub }} \times p$ the distance between two modulation elements. As for the metasurface array, when we calculate the scattering field, we have to consider the spatial phase difference $e^{j \beta N_{\text {sub }} p \sin \theta}$ between the I and Q paths.

As shown in Fig. $1, I_{n}(t)$ and $Q_{n}(t)$ are the time sequences of the paths, which, in turn, are obtained combining the time sequences of the unit-cells $i_{n}(t)$ and $q_{n}(t)$ as:

$$
\begin{aligned}
& I_{n}(t)=\sum_{i=1}^{N_{\text {suth }}} i_{n}(t) e^{j \beta(i-1) p \sin \theta} \\
& Q_{n}(t)=\sum_{i=1}^{N_{\text {sub }}} q_{n}(t) e^{j \beta(i-1) p \sin \theta}
\end{aligned}
$$

where $i_{n}(t)$ and $q_{n}(t)$ are given by:

$$
\begin{gathered}
i_{n}(t)=\left\{\begin{array}{l}
1, t_{1 n} \leq t / T_{p} \leq t_{1 n}+\tau \\
-1, t_{2 n} \leq t / T_{p} \leq t_{2 n}+\tau \\
0, \quad \text { others }
\end{array}\right. \\
q_{n}(t)=\left\{\begin{array}{l}
-1, t_{1 n}^{\prime} \leq t / T_{p} \leq t_{1 n}^{\prime}+\tau \\
1, t_{2 n}^{\prime} \leq t / T_{p} \leq t_{2 n}^{\prime}+\tau \\
0, \quad \text { others }
\end{array}\right.
\end{gathered}
$$

being $T_{p}$ the modulation period, which corresponds to the $+1 s t$ or $-1 s t$ order harmonic frequency shift. Therefore, the time sequences of the I/Q paths pair constituting the modulation element can be expressed as:

$$
\begin{aligned}
I_{n}(t)+j e^{j \beta N_{s u b} p \sin \theta} Q_{n}(t) \\
=\sum_{i=1}^{N s u b}\left[i_{n}(t)+j e^{j \beta N_{s u b p} p \sin \theta} q_{n}(t)\right] e^{j \beta(i-1) p \sin \theta}
\end{aligned}
$$

The periodic time sequences of the unit-cells are decomposed by using the Fourier series to highlight the contribution of the different frequency harmonics as:

$$
\begin{aligned}
i_{n}(t) & +j e^{j \beta N_{s u b} p \sin \theta} q_{n}(t) \\
& =\sum_{h=-\infty}^{+\infty}\left(a_{h n}+a_{h n}^{\prime}\right) e^{j 2 \pi h f_{p} t}, h=0, \pm 1, \ldots \pm \infty
\end{aligned}
$$

where $h$ is the order of the harmonics, $n$ refers to the $n t h$ "modulation element", $A_{h n}=a_{h n}+a_{h n}^{\prime}$ are the coefficients of the Fourier series, whose expression is evaluated in (9).

In order to only keep the first order harmonic and suppress the unwanted higher order ones, we have to analyze each term of the coefficient $A_{h n}$ in (9) and find the corresponding conditions. Considering the term:

$$
\begin{aligned}
& \sin \left(h \pi\left(t_{2 n}-t_{1 n}\right)\right) e^{-j h \pi\left(t_{2 n}+t_{1 n}+\tau\right)} e^{j \pi / 2} \\
& \quad+\sin \left(h \pi\left(t_{2 n}^{\prime}-t_{1 n}^{\prime}\right)\right) e^{-j h \pi\left(t_{2 n}+t_{1 n}+\tau\right)} e^{j \beta N_{s u b} p \sin \theta}
\end{aligned}
$$

when $t_{2 n}-t_{1 n}=t_{2 n}^{\prime}-t_{1 n}^{\prime}=1 / 2$, all the even order harmonics are suppressed $\left|A_{h n}\right|=0\{h=2 k, k= \pm 1, \ldots \pm \infty\}$. For what concerns the term $\frac{2}{h \pi} \sin (h \pi \tau)$, when $\tau=1 / 3$, the $h$ th $(h=3 \mathrm{k}$, $\mathrm{k} \in \mathbb{Z})$ 


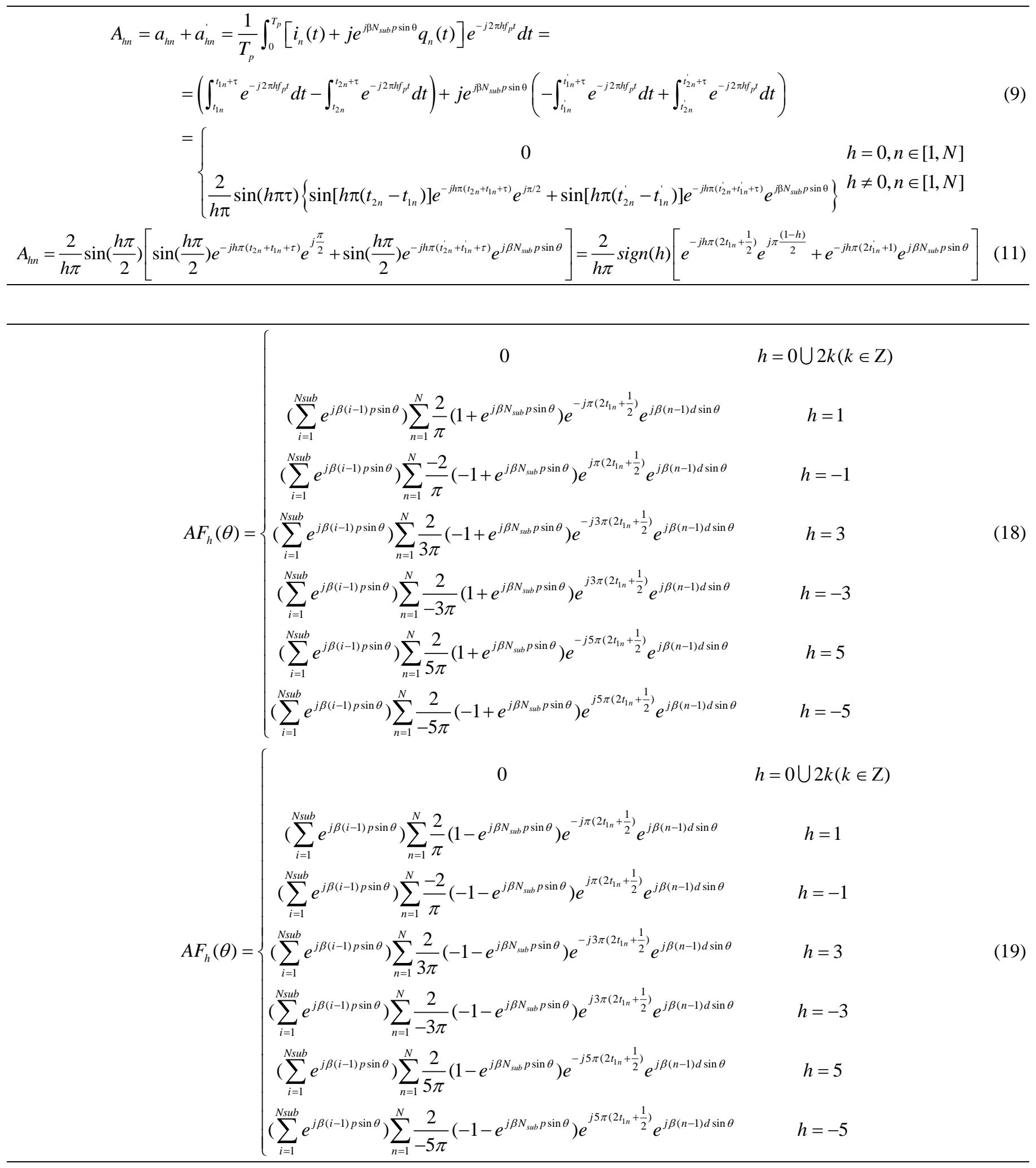


harmonics can be suppressed, $\left|A_{h n}\right|=0\{h=3 k, k= \pm 1, \ldots \pm \infty\}$. Ideally, this case results in the highest modulation efficiency. However, it is difficult to design a multi-functional unit-cell exhibiting the 2-bit phases and an absorption state to meet the required time sequences.

To simplify the implementation, we choose $\tau=1 / 2$. This would cause the presence of the $h t h(h=3 \mathrm{k}, \mathrm{k} \in \mathbb{Z})$ order odd harmonics, affecting, thus, the modulation efficiency. However, as will be demonstrated later by the numerical results, this choice leads to acceptable performances for the metasurface.

By substituting $t_{2 n}-t_{1 n}=t_{2 n}^{\prime}-t_{1 n}^{\prime}=1 / 2$ and $\tau=1 / 2$ into (9), we obtain formula (11). The coefficients of the first order harmonics are:

$$
\begin{aligned}
& A_{1 n}=\frac{2}{\pi}\left[e^{-j \pi\left(2 t_{1 n}+\frac{1}{2}\right)}+e^{-j \pi\left(2 t_{1 n}^{\prime}+1\right)} e^{j \beta N_{\text {sub }} p \sin \theta}\right] \\
& A_{-1 n}=\frac{2}{\pi}\left[e^{j \pi\left(2 t_{1 n}+\frac{1}{2}\right)}-e^{-j \pi\left(2 t_{1 n}^{\prime}+1\right)} e^{j \beta N_{\text {sub }} p \sin \theta}\right]
\end{aligned}
$$

If we aim at designing the metasurface to achieve a positive frequency shift (blue shift), only the $+1 s t$ order harmonic is retained, and the -1 st order harmonic must be suppressed. This can be obtained at $\theta=0$ (the specular reflection direction) for $t_{1 n}^{\prime}=t_{1 n}-1 / 4$ and $\left|A_{-1 n}\right|=0$, resulting in the largest amplitude for $A_{1 n}$. With this assumption, the Fourier coefficients for each harmonic are given by:

$$
A_{h n}=\frac{2}{h \pi} \operatorname{sign}(h)\left[e^{-j h \pi\left(2 t_{1 n}+\frac{1}{2}\right)}\left(e^{j \pi \frac{1-h}{2}}+e^{j \beta N_{\text {sub }} p \sin \theta}\right)\right]
$$

As noted in [42], the Fourier coefficients of the harmonics in (13) can be also expressed by using the equivalent amplitude and phase. As a result, the field scattered by a single modulation element of the metasurface can be written as:

$$
\begin{aligned}
& a f_{h n}(\theta)= \sum_{i=1}^{N s u b}\left[i_{n}(t)+j e^{j \beta N_{\text {sub }} p \sin \theta} q_{n}(t)\right] e^{j \beta(i-1) p \sin \theta} \\
&= \frac{2}{h \pi} \operatorname{sign}(h)\left(e^{j \pi \frac{1-h}{2}}+e^{j \beta N_{\text {sub }} p \sin \theta}\right) \\
& \cdot e^{-j h \pi\left(2 t_{1 n}+\frac{1}{2}\right)} \sum_{i=1}^{N s u b} e^{j \beta(i-1) p \sin \theta}
\end{aligned}
$$

Similarly, for the case of a negative frequency shift (red shift), only $-1 s t$ order harmonic is retained, and the $+1 s t$ order harmonic is suppressed. In this case, with $t_{1 n}^{\prime}=t_{1 n}+1 / 4$ and $\left|A_{1 n}\right|=0, A_{-1 n}$ has the largest amplitude and the Fourier coefficients of the harmonics are given by:

$$
A_{h n}=\frac{2}{h \pi} \operatorname{sign}(h)\left[e^{-j h \pi\left(2 t_{1 n}+\frac{1}{2}\right)}\left(e^{j \pi \frac{1-h}{2}}+e^{-j h \pi} e^{j \beta N_{s u b} p \sin \theta}\right)\right]
$$

Accordingly, the field scattered by the individual modulation element is given by:

$$
\begin{array}{r}
a f_{h n}(\theta)=\sum_{i=1}^{N s u b}\left[i_{n}(t)+j e^{j \beta N_{s u b} p \sin \theta} q_{n}(t)\right] e^{j \beta(i-1) p \sin \theta} \\
=\frac{2}{h \pi} \operatorname{sign}(h)\left(e^{j \pi \frac{1-h}{2}}+e^{-j h \pi} e^{j \beta N_{s u b} p \sin \theta}\right) \\
\cdot e^{-j h \pi\left(2 t_{1 n}+\frac{1}{2}\right)} \sum_{i=1}^{N s u b} e^{j \beta(i-1) p \sin \theta}
\end{array}
$$

Besides, as to the $h t h\{h=(4 \mathrm{k}-1), \mathrm{k} \in \mathbb{Z}\}$ harmonics, we have discussed the harmonics corresponding to $\mathrm{k}=0$ and $\mathrm{k}=1$, while for larger values of $\mathrm{h}$, the amplitudes of the corresponding harmonics can be neglected, being more than $16.90 \mathrm{~dB}$ lower than the first order harmonic, as shown in eq.(13) and (15).

\section{Analytical model of the I/Q space-time metasurface}

The field scattered by the entire array is the sum of the fields scattered by all the modulation elements and, thus, it reads:

$$
A F_{h}(\theta)=\sum_{n=1}^{N} a f_{h n}(\theta) e^{j \beta(n-1) d \sin \theta}
$$

For the blue shift, the harmonics resulting from (17) are listed in (18), while for the red shift, the resulting harmonics are listed in (19).

Based on the above derivation, namely $t_{2 n}-t_{1 n}=t_{2 n}^{\prime}-t_{1 n}^{\prime}=$ $1 / 2$ and $\tau=1 / 2$, for blue shift $t_{1 n}^{\prime}=t_{1 n}-1 / 4$, and for red shift $t_{1 n}^{\prime}=t_{1 n}+1 / 4$, the time variations of the phase $\phi(t)$ and $\phi^{\prime}(t)$ corresponding to the time sequences $i(t)$ and $q(t)$, respectively, are shown in Fig. 3.

Then we explore here, in the non-specular reflection observation directions, the opposite first order harmonic can be easily controlled by changing the dimension of I/Q paths, while the objective first order harmonic remains unchanged. Thus, the field scattered by the proposed metasurface depends on the dimension of the paths, which should introduce a spatial modulation. Let us first consider the $+1 s t$ or $-1 s t$ order harmonics. Without loss of generality, an $8 \times 16$ metasurface is considered with $N_{\text {sub }}$ unit-cells in each path. For the blue shift, the scattered field of the \pm 1 st harmonics is given by:

$$
\begin{aligned}
& \left|A F_{+1}\right|=\frac{2}{\pi}\left|\sum_{i=1}^{N_{\text {sub }}} e^{j \beta(i-1) p \sin \theta} \sum_{n=1}^{8 / N_{\text {sub }}}\left(1+e^{j \beta N_{\text {sub }} p \sin \theta}\right) e^{j \beta(n-1) 2 N_{\text {sub }} p \sin \theta}\right| \\
& \left|A F_{-1}\right|=\frac{2}{\pi}\left|\sum_{i=1}^{N_{\text {sub }}} e^{j \beta(i-1) p \sin \theta} \sum_{n=1}^{8 / N_{\text {sub }}}\left(-1+e^{j \beta N_{\text {sub }} p \sin \theta}\right) e^{j \beta(n-1) 2 N_{\text {sub }} p \sin \theta}\right|
\end{aligned}
$$

Calculating the summations in (20), we obtain:

$$
\begin{aligned}
\left|A F_{+1}\right| & =\frac{2}{\pi}\left|\frac{\sin \left(\frac{\beta N_{\text {sub }} p \sin \theta}{2}\right)}{\sin \left(\frac{\beta p \sin \theta}{2}\right)}\right|\left|\frac{\sin (\beta 8 p \sin \theta)}{\sin \left(\frac{\beta N_{s u b} p \sin \theta}{2}\right)}\right| \\
& =\frac{2}{\pi}\left|\frac{\sin (\beta 8 p \sin \theta)}{\sin \left(\frac{\beta p \sin \theta}{2}\right)}\right|
\end{aligned}
$$




$$
\begin{aligned}
\left|A F_{-1}\right| & =\frac{4}{\pi}\left|\frac{\sin \left(\frac{\beta N_{\text {sub }} p \sin \theta}{2}\right)}{\sin \left(\frac{\beta p \sin \theta}{2}\right)}\right|\left|\sin \frac{\beta N_{\text {sub }} p \sin \theta}{2}\right|\left|\frac{\sin (\beta 8 p \sin \theta)}{\sin \left(\beta N_{\text {sub }} p \sin \theta\right)}\right| \\
& =\frac{4}{\pi}\left|\frac{\sin ^{2}\left(\frac{\beta N_{\text {sub }} p \sin \theta}{2}\right)}{\sin \left(\frac{\beta p \sin \theta}{2}\right)}\right|\left|\frac{\sin (\beta 8 p \sin \theta)}{2 \sin \left(\frac{\beta N_{\text {sub }} p \sin \theta}{2}\right) \cos \left(\frac{\beta N_{\text {sub }} p \sin \theta}{2}\right)}\right| \\
& =\frac{2}{\pi}\left|\tan \left(\frac{\beta N_{\text {sub }} p \sin \theta}{2}\right)\right| \frac{\sin (\beta 8 p \sin \theta)}{\sin \left(\frac{\beta p \sin \theta}{2}\right)} \mid
\end{aligned}
$$

In (20a), we recognize that the summation in the scattered field of the +1 st order harmonic is a geometric series and, thus, it is independent of $N_{\text {sub }}$ as in (21a). In contrast, in (20b), the summation in the scattered field of the - 1 st order harmonic is an interleaved geometric series and, thus, it depends on $N_{\text {sub }}$ as in (21b). Similarly, by using (18), we find that the scattering fields of the $-3 r d$ and +5 th order harmonics are also geometric series, so that they do not depend on the path dimension, as it happens for the +1 st order harmonic. Instead, being the fields scattered by the $+3 r d$ and -5 th order harmonics interleaved geometric series, they can be modulated by properly choosing the path dimension, as for the $-1 s t$ order harmonic. To sum up, when changing the dimension of the I/Q paths, the opposite first order harmonics can be easily controlled by changing the dimension of the I/Q paths, keeping the objective first order harmonic unchanged. This space-time modulated character of the objective and its opposite first order harmonics would provide new approaches for space- and frequency-division multiplexing or secure communications [52].

Finally, in order to check the scanning properties of the beam scattered by the metasurface, let us consider the $+1 s t$ order harmonic (similar considerations hold for the -1 st order one). From (14) and (17), we observe that some terms of the expression do not depend on $n$ and some others depend on $n$ and, thus, determine the superposition of the fields scattered by the "modulation elements" and, in turn, are responsible for the beam scanning. The term $e^{-j \pi\left(2 t_{1 n}+\frac{1}{2}\right)}$ is determined by the start time of the first periodic time sequence of the $n t h$ "modulation element": when the start time of the periodic time sequence varies within a period $t_{1 n} \in[-1 / 4,3 / 4]$, the corresponding phase $-\pi\left(2 t_{1 n}+1 / 2\right)$ covers the $2 \pi$ range. Therefore, by properly designing the start time of the sequences $t_{1 n}$, it is possible to achieve high efficiency beam scanning of the +1 st order harmonic. Thus, to achieve a blue shift with pre-designed scanning angle $\theta=\theta_{\text {scan }}$, using the other term $e^{j \beta(n-1) d \sin \theta}$, we find the expression for $t_{1 n}$ as:

$$
t_{1 n}=\frac{1}{2}\left[\frac{\beta(n-1) d \sin \theta_{\text {scan }}}{\pi}-\frac{1}{2}\right]
$$

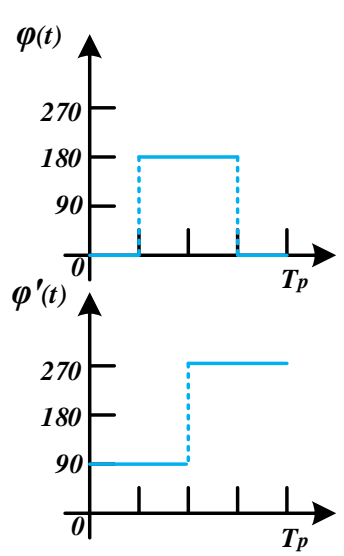

(a)

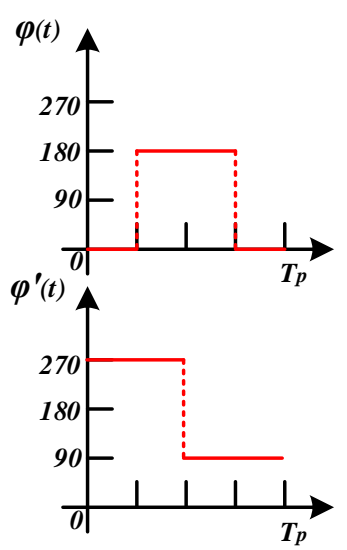

(b)
Fig. 3. The time-varying phases to achieve a (a) blue shift and (b) red shift in a modulation period. $\varphi(t)$ and $\varphi^{\prime}(t)$ correspond to $i_{n}(t)$ and $q_{n}(t)$, respectively.

\section{I/Q SPACE-TIME-MODULATED METASURFACE IMPELEMENTATION WITH 2-BIT UNIT CELLS}

\section{A. Space-time-modulated metasurface implementation}

To implement the space-time modulated metasurface, the 2-bit unit-cell originally proposed in [51] for other purposes is employed to match the time sequences reported in Fig. 3. The unit-cell with small electrical size is chosen to achieve the functionality of manipulating the opposite first order harmonic frequency by changing the dimension of I/Q paths. Thus, large range of I/Q paths dimensions composed of smaller unit-cells would produce diverse modulation. The unit-cell has been designed to exhibit the 2-bit phase response at $3.15 \mathrm{GHz}$ with a reflection amplitude larger than 0.9. Four different phase responses, denoted as " 0 ", " $\pi / 2$ ", " $\pi$ ", " $3 / 2 \pi$ " can be obtained by switching the ON/OFF states of three PIN diodes.

Considering the case of the blue shift, the corresponding implemented metasurface is shown in Fig. 5. According to the derived time varying phases of the modulation elements, it can be seen that the states of the entire array have been switched four times in a modulation period, that is, a quarter of a period corresponds to a phase state of the modulation element. The time variations of the reflection phases for the two I/Q paths constituting the modulation element are expressed as:

$$
\left\{\varphi(t), \varphi^{\prime}(t)\right\}=\left\{\begin{array}{l}
\{0, \pi / 2\}, t \in\left[0, \frac{T_{p}}{4}\right) \\
\{\pi, \pi / 2\}, t \in\left[\frac{T_{p}}{4}, \frac{T_{p}}{2}\right) \\
\{\pi, 3 \pi / 2\}, t \in\left[\frac{T_{p}}{2}, \frac{3 T_{p}}{4}\right) \\
\{0,3 \pi / 2\}, t \in\left[\frac{3 T_{p}}{4}, T_{p}\right)
\end{array}\right.
$$




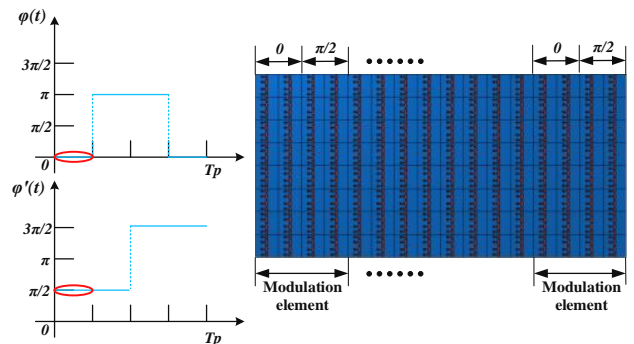

(a)
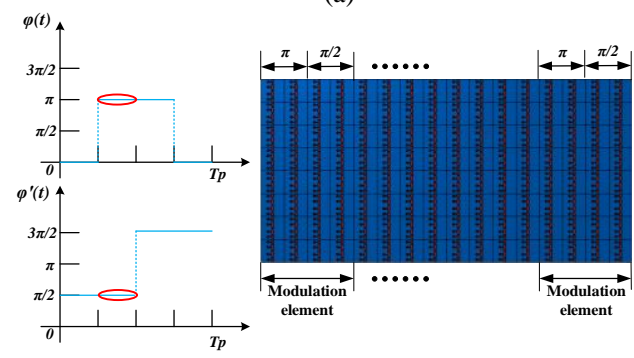

(b)

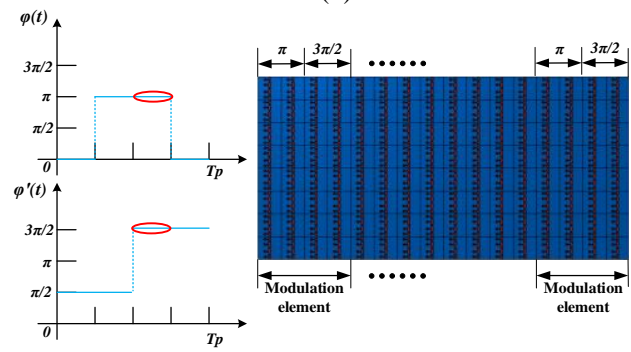

(c)

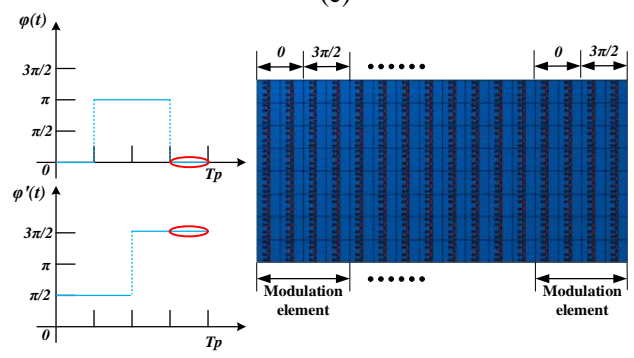

(d)

Fig. 5. The four modulation schemes of the metasurface for each quarter of the period of the time sequence to achieve a blue shift. Phase states within (a) the first quarter of the period (" 0 " and " $\pi$ "), (b) the second quarter of the period (" $\pi$ " and " $\pi / 2$ "), (c) the third quarter of the period ( " $\pi$ " and " $3 \pi / 2$ "), (d) the fourth quarter of the period (" 0 " and " $3 \pi / 2$ ").

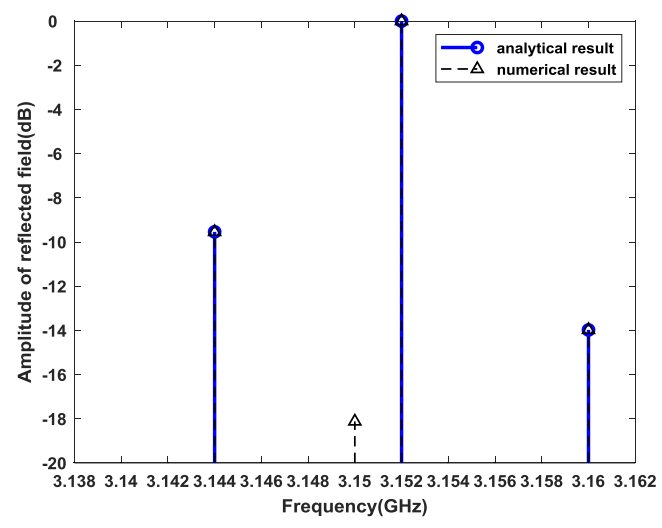

(a)

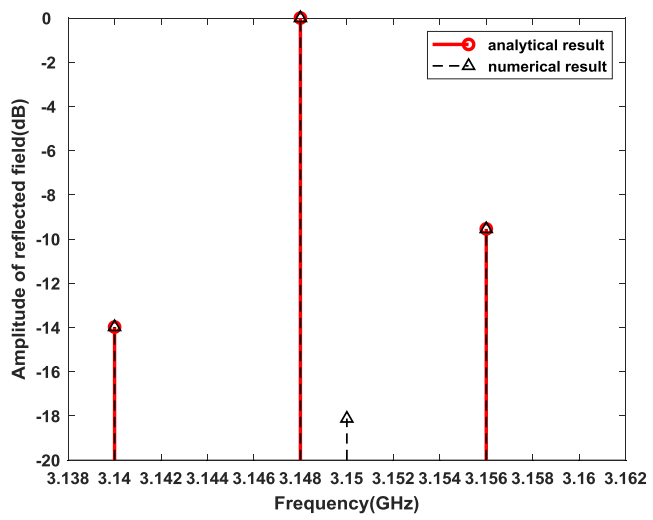

(b)

Fig. 6. Normalized reflection spectrum analytically calculated through the I/Q modulation formulas and numerically computed through time-domain simulations of the (a) blue and (b) red shifted reflected electromagnetic fields at $\theta=0$.

For the simulation, we used a similar procedure as in [29], based on a co-simulation between CST Studio Suite and Advanced Design System (ADS). In [30], however, being the time sequences of all the unit-cells the same, the authors simulated the frequency spectrum of the reflected field by considering a single unit-cell with periodic boundary conditions. In our design, instead, the reflection responses of the two I/Q paths in the modulation element are different. So, we considered the diode equivalent circuit and the phase states of the metasurface shown in Fig. 5, and performed the frequency domain simulations. Then, we have used CST to compute the field reflected by the array within each quarter of the period and combined such results to analyze the corresponding frequency spectrum. In particular, we have used the CST post-processing tools to perform the Fourier analysis of the reflected field time sequences needed to eventually obtain the frequency spectra.

The case of the red shift can be studied in a similar way, starting with the different time-varying phases shown in Fig. 3(b).

\section{RESULTS AND DISCUSSIONS}

\section{A. Blue and red shifts in the specular reflection direction}

In order to demonstrate the blue and red shifts using the proposed I/Q space-time-modulated metasurface, a time-domain simulation is implemented considering an $8 \times 16$ array. The designed frequency shift is $f_{p}=1 / T_{p}=2 \mathrm{MHz}$ and the number of cells for each path $N s u b=8$. The simulated frequency spectra of the normal reflected fields are shown in Fig. 6. An excellent agreement of the frequency shift can be found between the simulated and the analytical results obtained from (18). Besides the $\pm 3 r d$ order harmonics, as noted in Sec. II-B, there is the fundamental frequency component at 3.15 $\mathrm{GHz}$, which is due to the imperfect 2-bit phase of the designed metasurface unit-cell. As a result, the modulation efficiency would be, in turn, affected. However, as shown in Fig. 6, the amplitude of the unwanted fundamental frequency component is $18.05 \mathrm{~dB}$ lower than the ones of the desired \pm 1 st order 
harmonics, thus the influence on the frequency shift can be considered as negligible.

For the blue shift, the amplitude of the +1 st order harmonic is $9.54 \mathrm{~dB}$ higher than the one of the $-3 r d$ order harmonic and $13.98 \mathrm{~dB}$ higher than the one of the +5 th order harmonic, which results in a higher modulation efficiency.

For the red shift, the same modulation efficiency is found for the -1 st order harmonic.

From these results, it is clear that the highest modulation efficiency can be obtained by matching in the most accurate way possible the 2-bit phase. To this end, the unit-cell should exhibit a wide angle response and reduced mutual coupling among the elements. Theoretically, if we could design a multi-functional metasurface with both 2-bit phases and absorption states, then we would completely eliminate the $h t h$ $(h=3 \mathrm{k}, \mathrm{k} \in \mathbb{Z})$ order odd harmonics, in agreement with the theory presented in Sec. II-B. Ideally, when $\tau=1 / 3$, the designed I/Q modulation model can achieve the highest modulation efficiency, being the amplitude of the first order harmonic $13.98 \mathrm{~dB}$ higher than the amplitude of any other unwanted harmonic.

Besides, as for $(4 k-1)$ th $(\mathrm{k} \in \mathbb{Z})$ harmonics, when $\mathrm{k}=0$ and $\mathrm{k}$ $=1$, we have discuss the corresponding harmonics, and if $h$ becomes larger, the corresponding harmonics is more than 16.90dB difference from the first order harmonic. So they can be neglected.

\section{B. Opposite first order harmonic modulation by the dimension of I/Q paths}

In order to analyze the opposite harmonic modulation performances with the variation of the I/Q paths dimensions in the non-specular reflection directions, we simulate a metasurface for blue shift case with $8 \times 16$ unit-cells imparting a frequency shift $f_{p}=1 / T_{p}=2 \mathrm{MHz}$ with a different number of the unit-cells in the path, namely $N_{\text {sub }}=2,4$, and 8 . The analytical (evaluated through (21)) and full wave simulation results are shown in Fig. 7. As expected, in the non-specular reflection direction, with the increase of the I/Q paths dimension, the fields of the $-1 s t,+3 r d$ and $-5 t h$ order harmonics are modulated, while the scattering fields of the $+1 s t,-3 r d,+5 t h$ order harmonics maintain unchanged. And $-1 s t,+3 r d,-5 t h$ order harmonics have the same scattering patterns but with constant difference according to eq. (18). $+1 s t,-3 r d,+5$ th order harmonics also have the same relation between each other.

As to this functionality, we can assume a wireless communication scenario. In the specular reflection direction, the +1 st order harmonic is unchanged resulting, thus, in a constant transmission. In the other directions, instead, by changing the dimension of the I/Q paths, we can select the communication directions using the $-1 s t$ order harmonic. Combining this with the red shift case, a simultaneous BFSK (Binary Frequency Shift Keying) transmission to multi-point communication with reverse frequency coding can be performed. This unique functionality may provide new approaches for space- and frequency-division multiplexing communications .

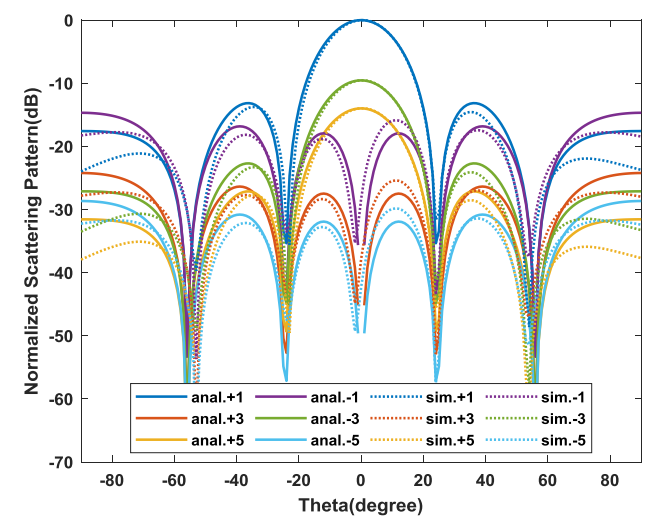

(a)

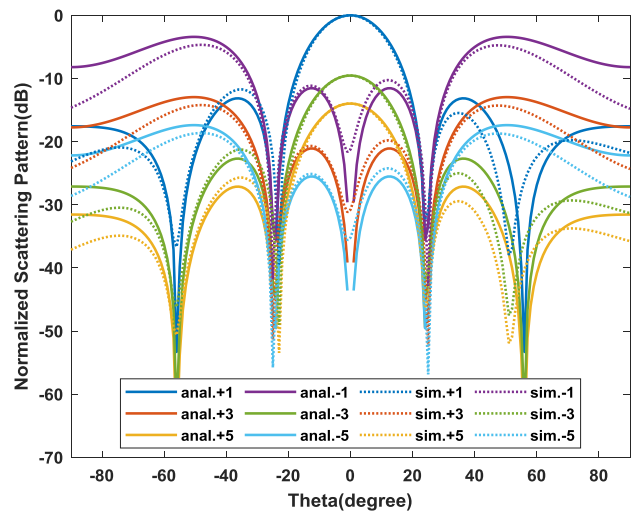

(b)

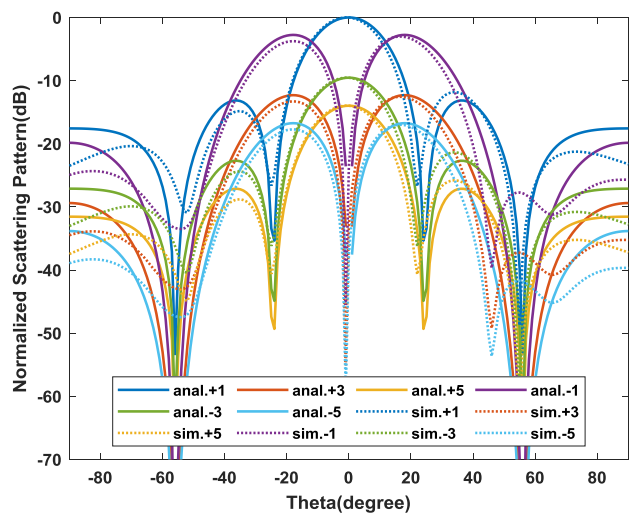

(c)

Fig. 7. Normalized scattering fields for the blue shift case: comparison between analytical (anal.) and full wave simulations (sim.) when (a) $N_{\text {sub }}=2$, (b) $N_{\text {sub }}=4$, and (c) $N_{\text {sub }}=8$.

\section{C. + 1st or -1 st order harmonic beam scanning}

In order to reflect the beam back to a specific scanning angle, the needed analytical start time is derived by using formula (22) and the corresponding scattering fields are calculated through (18). Considering the example of the blue shift, in order to achieve a narrower beam and low -1 st order harmonic lobes, we have designed a metasurface with $8 \times 32$ unit-cells imparting a frequency shift $f_{p}=1 / T_{p}=2 \mathrm{MHz}$ with $N s u b=2$ unit-cells for each path. The analytical results for the +1 st order harmonic beam scanning are shown in Fig. 8. At the 
design beam pointing angles, the resulting isolation of the $+1 s t$ order harmonic from other harmonics is beyond $10 \mathrm{~dB}$.

The start times normalized to the time period $T_{p}$ for the blue shift beam pointing angles $0^{\circ}, 10^{\circ}$, and $20^{\circ}$, are shown in Fig. 9 . Similar periodic time series can be designed analytically for red shift case. It should be noted that the design beam scanning angle is only dependent on the normalized time period, while the frequency shift is determined by the time period. As a result, thus, the proposed I/Q space-time modulated metasurface can manipulate the reflected fields in both space and frequency domain simultaneously.

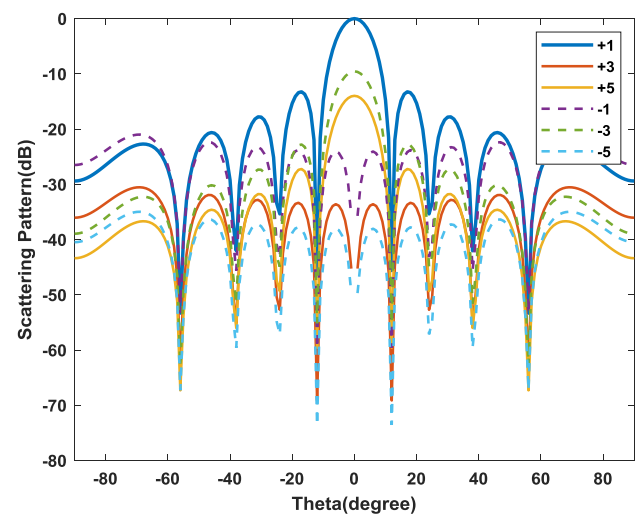

(a)

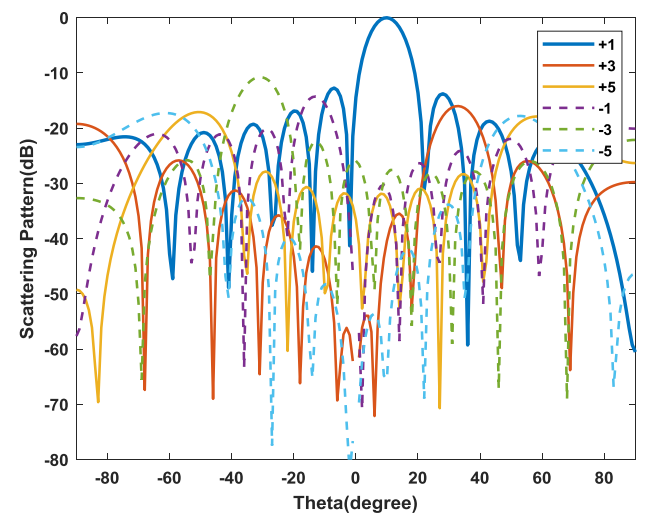

(b)

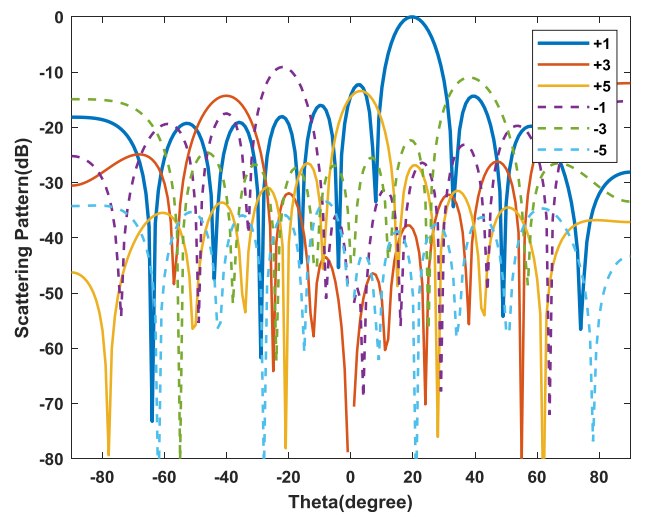

(c)

Fig. 8. Normalized scattered fields of the different harmonics for the blue shift case when the beams point at (a) $\theta_{\text {scan }}=0^{\circ}$, (b) $\theta_{\text {scan }}=10^{\circ}$, and (c) $\theta_{\text {scan }}=20^{\circ}$, respectively.

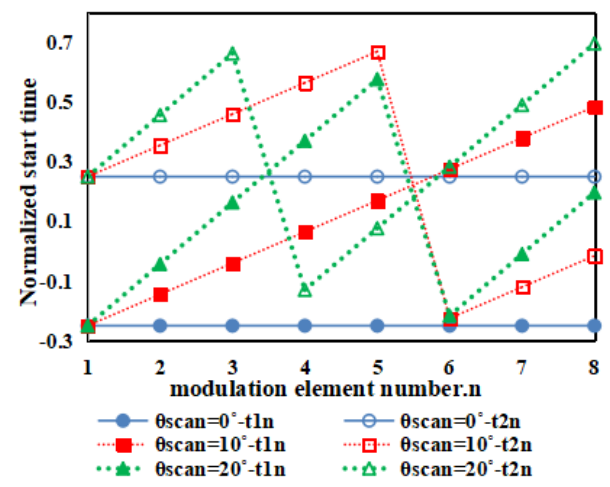

Fig. 9. Normalized start time with respect to the time period $T_{p}$ for scanning angle at $0^{\circ}, 10^{\circ}$, and $20^{\circ}$.

\section{CONCLUSION}

In this paper, a new design of an I/Q space-time-modulated metasurface is proposed. The periodic time sequences needed to achieve frequency shift and space-time beam scanning are derived. An I/Q space-time-modulated metasurface with 2-bit unit-cells is implemented and both analytical and numerical simulation results demonstrate the validity of the proposed solution. It should be noted that much higher efficiency can be achieved by using a multi-functional metasurface, where the unit-cells have 2-bit phases and an absorption state. The proposed I/Q space-time-modulated metasurfaces have potential applications in wireless communications, radar camouflaging.

\section{REFERENCES}

[1] S. Sun, Q. He, J. Hao, S. Xiao, and L. Zhou, "Electromagnetic metasurfaces: Physics and applications," Adv. Opt. Photon., vol. 11, no. 2, pp. 380-479, 2019.

[2] O. Quevedo-Teruel, H. Chen, A. Díaz-Rubio, et al. "Roadmap on metasurfaces," Journal of Optics, vol. 21. no. 7, Jul. 2019, Art. no. 073002.

[3] C. L. Holloway, E. F. Kuester, J. A. Gordon, J. O'Hara, J. Booth, and D. R. Smith, "An overview of the theory and applications of metasurfaces: The two-dimensional equivalents of metamaterials," IEEE Antennas Propag. Mag., vol. 54, no. 2, pp. 10-35, Apr. 2012

[4] X. Xia, Q. Wu, H. Wang, C. Yu, and W. Hong, "Wideband millimeter-wave microstrip reflectarray using dual-resonance unit cells," IEEE Antennas and Wireless Propag. Lett. vol. 16, pp. 4-7, Apr. 2016.

[5] G. Liu, H. Wang, J. S. Jiang, F. Xue, and M. Yi, “A high-efficiency transmitarray antenna using double split ring slot elements," IEEE Antennas and Wireless Propag. Lett., vol. 14, pp. 1415-1418, Mar. 2015.

[6] M. Chen, E. Abdo-Sánchez, A. Epstein, and G. V. Eleftheriades, "Theory, design, and experimental verification of a reflectionless bianisotropic Huygens' metasurface for wide-angle refraction," Phys. Rev. B, vol. 97, no. 12, p. 125433, Mar. 2018

[7] A. Epstein and G. V. Eleftheriades, "Synthesis of Passive Lossless Metasurfaces Using Auxiliary Fields for Reflectionless Beam Splitting and Perfect Reflection," Phys. Rev. Lett., vol. 117, no. 25, p. 256103, Dec. 2016

[8] A. Tennant and B. Chambers, "A single-layer tuneable microwave absorber using an active FSS," IEEE Microw. Wireless Compon. Lett., vol. 14, no. 1, pp. 46-47, Feb. 2004.

[9] L. Sun, H. Cheng, Y. Zhou, and J. Wang, "Broadband metamaterial absorber based on coupling resistive frequency selective surface," Optics Express, vol. 20, no. 4, pp. 4675-4680, Feb. 2012. 
[10] S. Zhou, X. Fang, M. Li, and R. S. Chen, "S/X dual-band real-time modulated frequency selective surface based absorber", Acta Physica Sinica, vol. 69, 20, 204101, 2020

[11] M. Khorasaninejad, W. T. Chen, R. C. Devlin, J. Oh, A. Y. Zhu, and F. Capasso, "Metalenses at visible wavelengths: Diffraction-limited focusing and subwavelength resolution imaging," Science, vol. 352, no. 6290, pp. 1190-1194, 2016.

[12] M. Li, S. Li, Y, Yu, X, Ni, and R. Chen, "Design of random and sparse metalens with matrix pencil method," Optics Express, vol. 26, no. 19, pp. 24702-24711, Sep. 2018

[13] M. Li, S. Li, L. K. Chin, Y. F. Yu, D. P. Tsai, and R. S. Chen, "Dual-layer achromatic metalens design with an effective Abbe number," Optics Express, vol. 28, no. 18, pp. 26041-26055, Aug. 2020.

[14] L. Li, Y. Shuang, Q. Ma, and T. J. Cui, "Intelligent metasurface imager and recognizer." Light Sci. Appl. vol. 8, no. 7, pp. 1-9, Oct. 2019.

[15] L. Li, H. Ruan, C. Liu, A. Alù, and T. J. Cui, "Machine-learning reprogrammable metasurface imager," Nat. Commun., vol. 10, no. 1, pp. 1-8, Mar. 2019

[16] H. C. Liu, B Yang., Q. Guo, J. H. Shi, C. Guan, G. X. Zheng, and S. Zhang, "Single-pixel computational ghost imaging with helicity-dependent metasurface hologram," Science advances, vol. 3, no. 9, Sep. 2017. Art. no. e1701477.

[17] A. Redo-Sanchez, B. Heshmat, A. Aghasi, S. Naqvi, M. Zhang, J. Romberg, and R. Raskar, "Terahertz time-gated spectral imaging for content extraction through layered structures,"Nat. Com., vol. 7, no. 1, pp 1-7, Sep. 2016.

[18] G. Minatti, M. Faenzi, E. Martini, F. Caminita, P. De Vita, D. González-Ovejero, and S. Maci, "Modulated metasurface antennas for space: Synthesis, analysis and realizations," IEEE Trans. Antennas Propag., vol. 63, no. 4, pp. 1288-1300, Apr. 2015.

[19] Y. Xie, W. Wang, H. Chen, A. Konneker, B. I. Popa, and S. A. Cummer, "Wavefront modulation and subwavelength diffractive acoustics with an acoustic metasurface," Nat. Commun., vol. 5, no. 1, pp. 1-5, Nov. 2014.

[20] K. Achouri, G. Lavigne, M. A. Salem, and C. Caloz, "Metasurface spatial processor for electromagnetic remote control," IEEE Trans. Antennas and Propag., vol. 64, no. 5, pp. 1759-1767, Mar. 2016.

[21] X. Wan, M. Q. Qi, T. Y. Chen, and T. J. Cui, "Field-programmable beam reconfiguring based on digitally-controlled coding metasurface," Sci. Rep., vol. 6, Feb. 2016, Art. no. 20663.

[22] C. Huang, B. Sun,W. Pan, X. G. Luo, "Dynamical beam manipulation based on 2-bit digitally-controlled coding metasurface," Sci. Rep., vol. 7, no. 1, pp. 1-8, Feb. 2017

[23] K. Chen, Y. Feng, F. Monticone, J. Zhao, B. Zhu, T. Jiang, and C. W. Qiu, "A reconfigurable active Huygens' metalens," Adv. Mater., vol. 29, no. 17, May 2017, Art. no. 1606422.

[24] W. Tang, J. Y. Dai, M. Chen, X. Li, Q. Cheng, S. Jin, K.-K. Wong, and T. J. Cui, "Programmable metasurface-based RF chain-free 8 PSK wireless transmitter," Electron. Lett., vol. 55, no. 7, pp. 417-420, Apr. 2019.

[25] J. Y. Dai, J. Zhao, Q. Cheng, and T. J. Cui, "Independent control of harmonic amplitudes and phases via a time-domain digital coding metasurface," Light Sci. Appl., vol. 7, no. 1, p. 90, Nov. 2018.

[26] L. Cong, P. Pitchappa, C. Lee, and R. Singh, "Active phase transition via loss engineering in a terahertz MEMS metamaterial," Adv. Mater., vol. 29, no. 26, Jul. 2017, Art. no. 1700733.

[27] Z. Miao, Q. Wu, X. Li, Q. He, K. Ding, Z. An, and L. Zhou, "Widely tunable terahertz phase modulation with gatecontrolled graphene metasurfaces," Phys. Rev. X, vol. 5, no. 4, 2015, Art. no. 041027.

[28] B. Y. Wei, W. Hu, Y. Ming, F. Xu, S. Rubin, J. G. Wang, and Y. Q. Lu, "Generating switchable and reconfigurable optical vortices via photo patterning of liquid crystals," Adv. ater., vol. 26, no. 10, pp. 1590-1595, Mar. 2014.

[29] D. Ramaccia, D. L. Sounas, A. Alù, A. Toscano, and F. Bilotti, "Phase-induced frequency conversion and Doppler effect with time-modulated metasurfaces," IEEE Trans. Antennas and Propag., vol. 68, no. 3, pp. 1607-1617, Mar. 2020.

[30] J. Zhao, X. Yang, J. Y. Dai, Q. Cheng, X. Li, N. H. Qi, and T. J. Cui, "Programmable time-domain digital-coding metasurface for non-linear harmonic manipulation and new wireless communication systems," National Science Review, vol. 6, no. 2, pp. 231-238, Nov. 2019.

[31] B. Liu, Y. He, S. W. Wong, and Y. Li, "Experimental demonstration of a time-domain digital-coding metasurface for a Doppler cloak," Optics Express, vol. 29, no. 2, pp. 740-750, Jan. 2021.
[32] J. Y. Dai, L. X. Yang, J. C. Ke, M. Z. Chen, W. Tang, X. Li and T. J. Cui. "High- Efficiency Synthesizer for Spatial Waves Based on Space- Time- Coding Digital Metasurface," Laser \& Photonics Reviews, vol. 14, no. 6, Art. no. 1900133, Jun. 2020.

[33] Z. Wu and A. Grbic. "Serrodyne frequency translation using time-modulated metasurfaces." IEEE Trans. Antennas and Propag., vol. 68 , no. 3, pp. 1599-1606. July 2019.

[34] N. Chamanara, S.Taravati, Z. L. Deck-Léger, ZL. Deck-Léger, and C. Caloz. "Optical isolation based on space-time engineered asymmetric photonic band gaps." Phys. Rev. B, vol. 96, no. 15, Art. no. 155409, Oct 2017.

[35] L. Zhang, X. Q. Chen, S. Liu, Q. Zhang, J. Zhao, J. Y. Dai, and T. J. Cui, "Space-time-coding digital metasurfaces," Nat. Commun., vol. 9, no. 1, pp. 1-11, Oct. 2018.

[36] Z. Wu, C. Scarborough, and A. Grbic. "Space-Time-Modulated Metasurfaces with Spatial Discretization: Free-Space N-Path Systems." Phys. Rev. Appl., vol. 14, no. 6, Art. no. 064060, Dec. 2020.

[37] D. Ramaccia, D. L. Sounas, A. V. Marini, A. Toscano, and F. Bilotti, "Electromagnetic isolation induced by time-varying metasurfaces: non-reciprocal bragg grating," IEEE Antennas and Wireless Propag. Lett., vol. 19, no. 11, pp. 1886-1890, May 2020.

[38] J. W. Zang, D. Correas-Serrano, J. T. S. Do, X. Liu, A. Alvarez-Melcon, and J. S. Gomez-Diaz, "Nonreciprocal wavefront engineering with time-modulated gradient metasurfaces," Phys. Rev. Appl., vol. 11, no. 5, Art. no. 054054, May 2019.

[39] A. E. Cardin, S. R. Silva, S. R. Vardeny, W. J. Padilla, A. Saxena, A. J. Taylor, and A. K. Azad. "Surface-wave-assisted nonreciprocity in spatio-temporally modulated metasurfaces," Nat. Commun., vol. 11, no. 1, pp. 1-9, Nov. 2020

[40] S, Taravati and G. V. Eleftheriades, "Full-duplex nonreciprocal beam steering by time-modulated phase-gradient metasurfaces," Phys. Rev. Appl. vol. 14, no. 1, Art. no. 014027, Jul. 2020.

[41] Y. Hadad, J. C. Soric, and A. Alu. "Breaking temporal symmetries for emission and absorption," Proceedings of the National Academy of Sciences, vol. 113, no. 13, pp. 3471-3475, Mar. 2016.

[42] L. Zhang, Z. X. Wang, R. W. Shao, J. L. Shen, X. Q. Chen, X. Wan, and T. J. Cui, "Dynamically realizing arbitrary multi-bit programmable phases using a 2-bit time-domain coding metasurface," IEEE Trans. Antennas and Propag., vol. 68, no. 4, pp. 2984-2292, Apr. 2020.

[43] J. Y. Dai, W. Tang, L. X. Yang, X. Li, M. Z. Chen, J. C. Ke, and T. J.Cui, "Realization of multi-modulation schemes for wireless communication by time-domain digital coding metasurface," IEEE Trans. Antennas and Propag., vol. 68, no. 3, pp. 1618-1627, Nov. 2019.

[44] W. Tang, X. Li, J. Y. Dai, S. Jin, Y. Zeng, Q. Cheng, and T. J. Cui,"Wireless communications with programmable metasurface: Transceiver design and experimental results," China Commun., vol. 16, no. 5, pp. 46-61, 2019.

[45] J. A. Hodge, K. V. Mishra, and A. I. Zaghloul. "Intelligent time-varying metasurface transceiver for index modulation in 6G wireless networks," IEEE Antennas and Wireless Propag. Lett., vol. 19, no. 11, pp. 1891-1895, Sep. 2020.

[46] W. Tang, M. Z. Chen, J. Y. Dai, Y. Zeng, X. Zhao, S. Jin, and T. J. Cui, "Wireless communications with programmable metasurface: New paradigms, opportunities, and challenges on transceiver design," IEEE Wireless Commun., vol. 27, no. 2, pp. 180-187, Apr. 2020.

[47] J. Y. Dai, W. K. Tang, J. Zhao, X. Li, Q. Cheng, J. C. Ke, and T. J. Cui, "Wireless communications through a simplified architecture based on time- domain digital coding metasurface," Adv. Mater., vol. 4, no. 7, Art. no. 1900044 , Feb. 2019.

[48] Q. Wu and R. Zhang. "Towards smart and reconfigurable environment: Intelligent reflecting surface aided wireless network," IEEE Commun. Mag., vol. 58, no. 1, pp. 106-112, Jan. 2019.

[49] X. Wang and C. Caloz, "Spread-spectrum selective camouflaging based on time-modulated metasurface," IEEE Trans. Antennas and Propag., vol. 69, no. 1, pp. 286-295, Jan. 2021.

[50] A. M. Yao, W. Wu, and D. G. Fang. "Single-side band time-modulated phased array," IEEE Trans. Antennas and Propag., vol. 63, no. 5, pp. 1957-1968, Feb. 2015.

[51] Y. Shuang, H. Zhao, W. Ji, T. J. Cui, and L. Li, "Programmable high-order OAM-carrying beams for direct-modulation wireless communications," IEEE Journal on Emerging and Selected Topics in Circuits and Systems, vol. 10, no. 1, pp. 29-37, Mar. 2020. 
[52] L. Zhang, M. Z. Chen, W. Tang, J. Y. Dai, T. J. Cui. "A wireless communication scheme based on space- and frequency-division multiplexing using digital metasurfaces," Nat. Electron., vol.4, no. 3, pp. 218-227, Mar. 2021. 